\title{
MANIFOLDS WITH A WEIGHTED POINCARÉ INEQUALITY
}

\author{
NGUYEN THAC DUNG AND CHIUNG-JUE ANNA SUNG
}

(Communicated by Lei $\mathrm{Ni}$ )

\begin{abstract}
We study complete manifolds satisfying a weighted Poincaré type property. We establish a splitting and vanishing theorem for $L^{2}$ harmonic forms provided that the weight function $\rho$ is of exponential growth of the distance function. Our theory generalizes the results of $\mathrm{Li}$-Wang, Lam and Chen-Sung.
\end{abstract}

\section{INTRODUCTION}

It is an interesting theme to understand the relations among the curvature, the topology and the function theorem on a Riemannian manifold. In the work of $\mathrm{Li}$ and Wang [L-W1] they proved a splitting type theorem for manifolds whose Ricci curvature is bounded from below by a negative multiple of the lower bound of the spectrum. In particular, their theorem generalized the work of Witten-Yau [W-Y], Cai-Galloway C-G] and X. Wang Wang on conformally compact manifolds to arbitrary complete manifolds. On the other hand, Li and Wang also proved the following vanishing theorem for $L^{2}$ integrable harmonic 1-forms on $M$ in [L-W1].

Theorem 1.1 (Li-Wang). Let $M$ be an m-dimensional complete Riemannian manifold with $\lambda_{1}(M)>0$ and

$$
\operatorname{Ric}_{M} \geq-\frac{m}{m-1} \lambda_{1}+\delta
$$

for some $\delta>0$. Then $H^{1}\left(L^{2}(M)\right)=0$, where $H^{1}\left(L^{2}(M)\right)$ denotes the space of $L^{2}$ integrable harmonic 1-forms on $M$.

Later, Lam [Lam] generalized Li-Wang's theory to manifolds satisfying a weighted Poincaré inequality.

Theorem 1.2 (Lam). Let $M$ be an m-dimensional complete Riemannian manifold satisfying a weighted Poincaré inequality with a nonnegative weight function $\rho(x)$. Assume that the Ricci curvature satisfies

$$
\operatorname{Ric}_{M}(x) \geq-\frac{m}{m-1} \rho(x)+\delta,
$$

for some $\delta>0$. Let $r(x, p)$ be the distance function from $x$ to some fixed point $p$, if $\rho(x)=O\left(r^{2-\alpha}(x, p)\right)$, for some $0<\alpha<2$. Then $H^{1}\left(L^{2}(M)\right)=0$.

Received by the editors June 8, 2012.

2010 Mathematics Subject Classification. Primary 53C40.

The first author was partially supported by the grant NAFOSTED 101.01-2011.13.

The second author was partially supported by the NSC. 
The main purpose of this article is to study structure theorems for manifolds satisfying a weighted Poincaré type property. In our theorems, a growth assumption is needed for the weighted function. Let us first recall some definitions.

Definition 1.3. Let $M^{m}$ be an $m$-dimensional complete Riemannian manifold. We say that $M^{m}$ satisfies a weighted Poincaré inequality (cf. [L-W2]) with a nonnegative weight function $\rho(x)$ if the inequality

$$
\int_{M} \rho(x) \phi^{2} \leq \int_{M}|\nabla \phi|^{2} \quad \text { for } \phi \in C_{0}^{\infty}(M)
$$

is valid for all compactly supported smooth functions $\phi \in C_{0}^{\infty}(M)$.

Definition 1.4. Let $M^{m}$ be an $m$-dimensional complete Riemannian manifold. We say that $M^{m}$ has property $\left(\mathcal{P}_{\rho}\right)$ if a weighted Poincaré inequality is valid on $M$ with some nonnegative weight function $\rho$. Moreover, the $\rho$-metric, defined by

$$
d s_{\rho}^{2}=\rho d s_{M}^{2},
$$

is complete.

Let $\lambda_{1}(M)$ denote the bottom spectrum of the Laplacian on $M$, namely,

$$
\lambda_{1}(M)=\inf \left\{\frac{\int_{M}|\nabla \phi|^{2}}{\int_{M} \phi^{2}}: \phi \in \mathcal{C}_{0}^{\infty}(M)\right\} .
$$

Then the variation principle for $\lambda_{1}(M)$ asserts the validity of the Poincaré inequality

$$
\lambda_{1}(M) \int_{M} \phi^{2} \leq \int_{M}|\nabla \phi|^{2}
$$

for all compactly supported functions $\phi \in C_{0}^{\infty}(M)$. In particular, if $\lambda_{1}(M)$ is assumed to be positive, then obviously $M$ possesses property $\left(\mathcal{P}_{\rho}\right)$ with $\rho(x)=$ $\lambda_{1}(M)$. The notion of property $\left(\mathcal{P}_{\rho}\right)$ may be viewed as a generalization of the assumption $\lambda_{1}(M)>0$.

For harmonic $p$-forms, let $C_{0}^{\infty}\left(\wedge^{p} M\right)$ denote the space of smooth $p$-forms with compact support on $M$. Then we define property $\left(\mathcal{P}_{p, \rho}\right)$ as follows.

Definition 1.5. Let $M^{m}$ be an $m$-dimensional complete Riemannian manifold. We say that $M^{m}$ has property $\left(\mathcal{P}_{p, \rho}\right)$ if a weighted $p$-Poincaré inequality is valid on $M$ with some non-negative weight function $\rho$, i.e.,

$$
\int_{M} \rho(x)|\phi|^{2} \leq \int_{M}|d \phi|^{2}+|\delta \phi|^{2} \quad \text { for } \phi \in C_{0}^{\infty}\left(\wedge^{p} M\right) .
$$

Moreover, the $\rho$-metric, defined by $d s_{\rho}^{2}=\rho d s_{M}^{2}$, is complete.

Note that if the greatest lower bound $\lambda_{1, p}$ of the $p$ spectrum satisfies $\lambda_{1, p}(M)>$ 0 , then $M$ has property $\left(\mathcal{P}_{p, \rho}\right)$ with the weight function $\rho(x)=\lambda_{1, p}(M)$. Hence the notion of property $\left(\mathcal{P}_{p, \rho}\right)$ can also be viewed as a generalization of the assumption that $\lambda_{1, p}(M)>0$.

Throughout this article, we use $H^{p}\left(L^{2}(M)\right)$ to denote the space of $L^{2}$ harmonic $p$-forms, and $r_{\rho}(x)$ the geodesic distance from some fixed point to $x$ with respect to the metric $d s_{\rho}^{2}$.

It is interesting to see that a similar theorem holds by relaxing the Ricci curvature bound condition with $\delta=0$. In this article, we show a result of [L-W1] and [Lam] to weighted $p$-Poincaré type property and establish a splitting and vanishing result for 
$L^{2}$ harmonic 1-forms provided that the weight function $\rho$ is of exponential growth of the distance function. The following theorem has been established.

Theorem 1.6. Let $M$ be an m-dimensional complete Riemannian manifold satisfying a $\mathcal{P}_{1, \rho}$ property with a nonnegative weight function $\rho(x)$. Assume that the Ricci curvature satisfies

$$
\operatorname{Ric}_{M}(x) \geq-\frac{m}{m-1} \rho(x)
$$

and $\rho \leq O\left(e^{2 r_{\rho}}\right)$, Then either

(1) $H^{1}\left(L^{2}(M)\right)=0$ or

(2) $\widetilde{M}=\mathbb{R} \times N$, where $\widetilde{M}$ is the universal cover of $M$ and $N$ is a manifold of dimension $(m-1)$.

On the other hand, we prove a similar result for $L^{2}$ harmonic $p$-forms on manifolds with property $\left(\mathcal{P}_{p, \rho}\right)$. The curvature operator $K_{p}$ on $M$ is assumed to satisfy the inequality

$$
K_{p} \geq-\frac{m-p+1}{m-p} \rho(x)
$$

for all $x \in M$. In this theorem, a growth assumption is also needed for the weight function $\rho$ in terms of the $\rho$-distance.

Theorem 1.7. Let $M$ be a complete noncompact manifold with property $\left(\mathcal{P}_{p, \rho}\right)$ for some nonnegative weight function $\rho$. Assume that the curvature operator $K_{p}$ on $M$ has the lower bound

$$
K_{p} \geq-\frac{m-p+1}{m-p} \rho(x)
$$

and $\rho \leq O\left(e^{2 r_{\rho}}\right)$. Suppose that $\omega$ is a harmonic $p$-form in $L^{2}(M)(p \geq 2)$. Then either

(1) $H^{p}\left(L^{2}(M)\right)=0$ or

(2) there exists a 1-form $\alpha$ with $\alpha \wedge \omega=0$ such that

$$
\nabla \omega=\alpha \otimes \omega-\frac{1}{m-p+1} \sum_{j=1}^{m} \theta^{j} \otimes\left(\theta^{j} \wedge i_{\alpha \# \omega}\right),
$$

where $\left\{\theta^{1,}, \ldots, \theta^{m}\right\}$ is an orthonormal basis for the cotangent bundle and $\alpha^{\#}$ is the dual vector of $\alpha$.

\section{Splitting And VAnishing theorems}

Let $\left\{e_{1}, \ldots, e_{m}\right\}$ be an orthonormal basis and harmonic 1-form $\omega=\sum_{i=1}^{m} \omega_{i} e_{i}$. It is well known that if $\omega$ is an $L^{2}$ harmonic 1-form, then it is both closed and co-closed:

$$
|\nabla \omega|^{2}=\sum_{i, j=1}^{m} w_{i j}^{2} \geq \sum_{j=1}^{m} w_{1 j}^{2}+\sum_{\alpha=2}^{m} w_{\alpha 1}^{2}+\sum_{\alpha=2}^{m} w_{\alpha \alpha}^{2} \geq \frac{m}{m-1} \sum_{j=1}^{m} w_{1 j}^{2} .
$$

We also know that $|\omega|^{2}|\nabla| \omega||^{2}=w_{1}^{2} \sum_{j=1}^{m} w_{1 j}^{2}$, hence

$$
|\nabla \omega|^{2} \geq \frac{m}{m-1}|\nabla| \omega||^{2}
$$


Therefore, $\omega$ satisfies the following Bochner type formula:

$$
|\omega| \Delta|\omega| \geq \operatorname{Ric}(\omega, \omega)+\frac{|\nabla| \omega||^{2}}{m-1} .
$$

By the lemma in Lam, we have an estimate for functions which satisfy the above Bochner type formula. For the sake of completeness, we restate the lemma in Lam as follows.

Lemma 2.1 ( $(\overline{\mathrm{Lam}})$. Let $b>-1$. Assume that $h$ is a nonnegative function and satisfies the differential inequality $h \Delta h \geq-a h^{2}+b|\nabla h|^{2}$ in the weak sense for some constant $a>0$. For any $\varepsilon>0$, we have the estimate

$$
(b(1-\varepsilon)+1) \int_{M}|\nabla(\phi h)|^{2} \leq\left\{b\left(\frac{1}{\varepsilon}-1\right)+1\right\} \int_{M} h^{2}|\nabla \phi|^{2}+a \int_{M} \phi^{2} h^{2}
$$

for any compactly supported smooth function $\phi \in \mathcal{C}_{0}^{\infty}(M)$. In addition, if

$$
\int_{B_{p}(R)} h^{2}=o\left(R^{2}\right)
$$

then

$$
\int_{M}|\nabla h|^{2} \leq \frac{a}{b+1} \int_{M} h^{2}
$$

In particular, $h$ has a finite Dirichlet integral if $h \in L^{2}(M)$.

Note that the results of Lemma 3.1 in Lam are still valid if $a=a(x)$ is a function of $x$. Hence, we have

$$
(b(1-\epsilon)+1) \int_{M}|\nabla(\varphi h)|^{2} \leq\left(b\left(\frac{1}{\epsilon}-1\right)+1\right) \int_{M} h^{2}|\nabla \varphi|^{2}+\int_{M} a(x) \varphi^{2} h^{2}
$$

and

$$
\int_{M}|\nabla h|^{2} \leq \frac{1}{b+1} \int_{M} a(x) h^{2} .
$$

In this section, the structure theorems are established for complete manifolds that satisfy a weighted Poincaré type inequality with weight function $\rho(x)$. The following theorem is given on a manifold with positive spectrum $\lambda_{1}(M)$; i.e. $M$ possesses property $\left(\mathcal{P}_{\rho}\right)$ with weight function $\rho=\lambda_{1}(M)$.

Theorem 2.2. Let $M$ be a complete Riemannian manifold of dimension $m \geq 3$. Suppose that $\lambda_{1}(M)>0$ and

$$
\operatorname{Ric}_{M} \geq-\frac{m}{m-1} \lambda_{1}(M)
$$

Then, either

(1) $H^{1}\left(L^{2}(M)\right)=0$ or

(2) $\widetilde{M}=\mathbb{R} \times N$, where $\widetilde{M}$ is the universal cover of $M$ and $N$ is a manifold of dimension $(m-1)$.

Proof. Let $\omega$ be an $L^{2}$ harmonic 1-form and $h=|\omega|$ the length of $\omega$. By the Bochner type formula (3) and the curvature assumption, we have

$$
h \Delta h \geq-\frac{m \lambda_{1}(M)}{m-1} h^{2}+\frac{1}{m-1}|\nabla h|^{2} .
$$


By Lemma 2.1 $h$ has a finite Dirichlet integral. Let $\phi$ be a cut-off function such that

$$
\phi= \begin{cases}1 & \text { on } B(R) \\ 0 & \text { on } M \backslash B(2 R)\end{cases}
$$

and $|\nabla \phi|^{2} \leq \frac{C}{R^{2}}$ on $B(2 R) \backslash B(R)$.

Now we consider the following cases.

Case $1\left(\lambda_{1}(M) \int_{M} h^{2}<\int_{M}|\nabla h|^{2}\right)$. Since $h$ has finite Dirichlet integral, we have

$$
\lim _{R \rightarrow \infty} \int_{B(2 R)} \phi^{2} h^{2}=\int_{M} h^{2} \text { and } \lim _{R \rightarrow \infty} \int_{B(2 R)}|\nabla(\phi h)|^{2}=\int_{M}|\nabla h|^{2} .
$$

Hence, there exists a positive number $\eta$ such that

$$
\left(\lambda_{1}(M)+\eta\right) \int_{M}(\phi h)^{2} \leq \int_{M}|\nabla(\phi h)|^{2} \quad \text { if } R \text { is large enough. }
$$

By Lemma 2.1, we have

$$
\begin{aligned}
&\left(\frac{1}{m-1}(1-\varepsilon)+1\right)\left(\lambda_{1}(M)+\eta\right) \int_{M} \phi^{2} h^{2} \leq\left(\frac{1}{m-1}(1-\varepsilon)+1\right) \int_{M}|\nabla(\phi h)|^{2} \\
& \leq \frac{m \lambda_{1}(M)}{m-1} \int_{M} \phi^{2} h^{2}+\left\{\frac{1}{m-1}\left(\frac{1}{\varepsilon}-1\right)+1\right\} \int_{M} h^{2}|\nabla \phi|^{2}
\end{aligned}
$$

for any $\varepsilon>0$ and any compactly supported smooth function $\phi \in \mathcal{C}_{0}^{\infty}(M)$ when $R$ is large. The above inequality yields

$$
\frac{\eta(1-\varepsilon)}{m-1} \int_{B(R)} h^{2} \leq \frac{\varepsilon \lambda_{1}(M)}{m-1} \int_{B(R)} h^{2}+\left\{\frac{1}{m-1}\left(\frac{1}{\varepsilon}-1\right)+1\right\} \frac{C}{R^{2}} \int_{B(2 R) \backslash B(R)} h^{2} .
$$

Since $h \in L^{2}(M)$, first let $R \rightarrow \infty$, then take $\varepsilon \rightarrow 0$. We conclude that $\int h^{2} \leq 0$. Thus, $h \equiv 0$, equivalently, $\omega \equiv 0$. This yields a contradiction, since $\lambda_{1}(M) \int_{M} h^{2}<$ $\int_{M}|\nabla h|^{2}$.

Case $2\left(\lambda_{1}(M) \int_{M} h^{2}=\int_{M}|\nabla h|^{2}\right)$.

Multiplying both sides of inequality (6) by $\phi$ and integrating it over $M$, the divergence theorem implies that

$$
-\int_{M}\langle\nabla(\phi h), \nabla h\rangle \geq-\frac{m \lambda_{1}(M)}{m-1} \int_{M} \phi h^{2}+\frac{1}{m-1} \int_{M} \phi|\nabla h|^{2} .
$$

Let $R \rightarrow \infty$ and use the fact that $h$ and $|\nabla h|$ are in $L^{2}(M)$ :

$$
-\int_{M}|\nabla h|^{2} \geq-\frac{m \lambda_{1}(M)}{m-1} \int_{M} h^{2}+\frac{1}{m-1} \int_{M}|\nabla h|^{2} .
$$

We have

$$
-\lambda_{1}(M) \int_{M} h^{2} \geq-\frac{m \lambda_{1}(M)}{m-1} \int_{M} h^{2}+\frac{1}{m-1} \int_{M}|\nabla h|^{2} .
$$


This implies

$$
\lambda_{1}(M) \int_{M} h^{2} \geq \int_{M}|\nabla h|^{2} .
$$

Hence equality (3) holds. In particular, the gradient of $\omega$ must be

$$
\left(w_{i j}\right)=\left(\begin{array}{ccccc}
-(n-1) \mu & 0 & 0 & \ldots & 0 \\
0 & \mu & 0 & \ldots & 0 \\
0 & 0 & \mu & \ldots & 0 \\
\vdots & \vdots & \vdots & \ddots & \vdots \\
0 & 0 & 0 & \ldots & \mu
\end{array}\right)
$$

if $\omega$ is harmonic.

Since the equality (6) holds,

$$
h \Delta h=-\frac{m \lambda_{1}(M)}{m-1} h^{2}+\frac{1}{m-1}|\nabla h|^{2} .
$$

Let $g=h^{\frac{m-2}{m-1}}$; we have

$$
-\frac{m(m-2) \lambda_{1}(M)}{(m-1)^{2}} g=\Delta g
$$

Now, suppose that the space of an $L^{2}$ harmonic 1-form is nontrivial, i.e., $H^{1}\left(L^{2}(M)\right) \neq 0$. Then by equation (7),

$H^{1}\left(L^{2}(M)\right) \subset\left\{\omega: \omega\right.$ is a harmonic 1-form: $\left.\Delta|\omega|^{\frac{m-2}{m-1}}=-\frac{m(m-2) \lambda_{1}(M)}{(m-1)^{2}}|\omega|^{\frac{m-2}{m-1}}\right\}$.

Next, we show that $\widetilde{M}$ is equal to $\mathbb{R} \times N$ if $\pi: \widetilde{M} \rightarrow M$ is a universal cover of $M$.

If $\omega$ is an $L^{2}$ harmonic 1-form, then $\omega$ is closed. Let $\widetilde{\omega}$ be the lifting of $\omega$ on $\widetilde{M}$ with respect to covering map $\pi$. Note that $\widetilde{\omega}$ is closed. Because $\widetilde{M}$ is simply connected, there exists a function $f$ on $\widetilde{M}$ such that $d f=\widetilde{\omega}$ and the Hessian matrix of $f$ is a diagonal matrix. In particular, the level set of $f$ and $|\nabla f|$ coincide. Let $N$ be a fixed level set of $f$ in $\widetilde{M}$. Since $|\nabla f| \neq 0, \widetilde{M}$ is equal to $\mathbb{R} \times N$.

Let $\left(x_{2}, \ldots, x_{m}\right)$ be a fixed point in $N$. The image of $\mathbb{R} \times\left\{x_{2}, \ldots, x_{m}\right\}$ via the covering map $\pi$ becomes a totally geodesic hypersurface of $M$.

This completes the proof of Theorem 2.2 .

Remark 2.3.

(1) Note that by equation (77) and Li-Wang's argument, we have that $M$ has only one end. Recall that an end of $M$ is an unbounded component of $M \backslash K$, where $K$ is a compact subset of $M$.

(2) If the Ricci curvature of $M$ satisfies $\operatorname{Ric}_{M} \geq-\frac{m}{m-1} \lambda_{1}(M)+\delta$ for some positive constant $\delta>0$, then equality (7) is no longer true and $H^{1}\left(L^{2}(M)\right)=0$. Hence Theorem 2.2 is a generalization of the work of Li-Wang [L-W1] and Lam Lam.

Next, we generalize the above result to show that manifold $M$ satisfies a weighted Poincaré type inequality with a nonconstant weight function. 
Theorem 2.4. Let $M$ be an $m$-dimensional complete Riemannian manifold satisfying a $\left(\mathcal{P}_{1, \rho}\right)$ property with a nonnegative weight function $\rho(x)$. Assume that the Ricci curvature satisfies

$$
\operatorname{Ric}_{M}(x) \geq-\frac{m}{m-1} \rho(x)
$$

and $\rho \leq O\left(e^{2 r_{\rho}}\right)$, Then either

(1) $H^{1}\left(L^{2}(M)\right)=0$ or

(2) $\widetilde{M}=\mathbb{R} \times N$, where $\widetilde{M}$ is the universal cover of $M$ and $N$ is a manifold of dimension $(m-1)$.

Proof. Suppose that $H^{1}\left(L^{2}(M)\right) \neq 0$. Let $\omega$ be an $L^{2}(M)$ harmonic 1-form and $h=|\omega|$. We will first prove that

$$
\int_{M} \rho|\omega|^{2}<\infty
$$

Indeed, $\rho \leq O\left(e^{2 r_{\rho}}\right)$ and by $|\omega| \in L^{2}(M)$, for any $R>0$, we obtain

$$
\int_{B_{\rho}(2 R) \backslash B_{\rho}(R)} \rho e^{-2 r_{\rho}}|\omega|^{2} \leq C
$$

for some constant $C$. By Lemma 2.1 in $[\mathrm{C}-\mathrm{S}]$,

$$
\int_{B_{\rho}(R+1) \backslash B_{\rho}(R)} \rho|\omega|^{2} \leq C e^{-2 R} .
$$

Hence,

$$
\begin{aligned}
\int_{B_{\rho}(2 R) \backslash B_{\rho}(R)} \rho|\omega|^{2} & \leq \sum_{r=[R]}^{2[R]+1} \int_{B_{\rho}(r+1) \backslash B_{\rho}(r)} \rho|\omega|^{2} \\
& \leq \sum_{r=[R]}^{2[R]+1} C e^{-2 r} \leq 2 C[R] e^{-2[R]}
\end{aligned}
$$

Since $\sum_{n=1}^{\infty}[R] 2^{n} e^{-2^{n}[R]}$ converges, we have

$$
\begin{aligned}
\int_{M} \rho|\omega|^{2} & =\int_{B(R)} \rho|\omega|^{2}+\sum_{n=1}^{\infty} \int_{B_{\rho}\left(2^{n} R\right) \backslash B_{\rho}\left(2^{n-1} R\right)} \rho|\omega|^{2} \\
& \leq \int_{B(R)} \rho|\omega|^{2}+C[R] \sum_{n=1}^{\infty} 2^{n} e^{-2^{n}[R]}<\infty .
\end{aligned}
$$

Now, by the Bochner type formula (3) and the curvature assumption,

$$
h \Delta h \geq-\frac{m \rho(x)}{m-1} h^{2}+\frac{1}{m-1}|\nabla h|^{2} .
$$

Note that in this formula, we have $a(x)=\frac{m \rho(x)}{m-1}, b=\frac{1}{m-1}$. By (5) and (8), $h$ has a finite Dirichlet integral. Let $\phi$ be a cut-off function such that

$$
\phi= \begin{cases}1, & \text { on } B(R), \\ 0, & \text { on } M \backslash B(2 R)\end{cases}
$$

and $|\nabla \phi|^{2} \leq \frac{C}{R^{2}}$ on $B(2 R) \backslash B(R)$. 
By a similar proof in Theorem 2.2 and inequality (5), we have

$$
\int_{M}|\nabla h|^{2} \leq \frac{1}{\frac{1}{m-1}+1} \int_{M} \frac{m \rho(x)}{m-1} h^{2}=\int_{M} \rho(x) h^{2} .
$$

However, if $M$ satisfies the property $\left(\mathcal{P}_{1, \rho}\right)$, then the weighted Poincaré inequality implies $\int_{M} \rho(x) h^{2}=\int_{M}|\nabla h|^{2}$. On the other hand, if we multiply both sides of (9) by $\phi$ and integrate over $M$, the divergence theorem implies that

$$
-\int_{M}\langle\nabla(\phi h), \nabla h\rangle \geq-\frac{m}{m-1} \int_{M} \phi \rho(x) h^{2}+\frac{1}{m-1} \int_{M} \phi|\nabla h|^{2} .
$$

Now, letting $R \rightarrow \infty$ and using the fact that $h \in L^{2}(M),|\nabla h| \in L^{2}(M)$, we have

$$
-\frac{m}{m-1} \int_{M} \rho(x) h^{2}+\frac{1}{m-1} \int_{M}|\nabla h|^{2} \leq-\int_{M}|\nabla h|^{2}
$$

and

$$
-\frac{m}{m-1} \int_{M} \rho(x) h^{2}+\frac{1}{m-1} \int_{M}|\nabla h|^{2} \leq-\int_{M} \rho(x) h^{2} .
$$

This implies

$$
\int_{M}|\nabla h|^{2} \leq \int_{M} \rho(x) h^{2} .
$$

So all preceding inequalities becomes equalities. In particular, the inequality (9) is an equality, namely,

$$
h \Delta h=-\frac{m \rho(x)}{m-1} h^{2}+\frac{1}{m-1}|\nabla h|^{2} .
$$

By a similar proof in Theorem 2.2, we conclude that either $H^{1}\left(L^{2}(M)\right)=0$ or $\widetilde{M}=\mathbb{R} \times N$, where $\widetilde{M}$ is the universal cover of $M$ and $N$ is a manifold of dimension $m-1$. The proof is complete.

Remark 2.5. If $\rho=O\left(r^{2-\alpha}\right)$ where $\alpha>0$ is given, then $d s_{\rho}^{2}=\rho d s_{M}^{2}=O\left(r^{2-\alpha} d s_{M}^{2}\right)$. We have

$$
\rho e^{-2 r_{\rho}}=O\left(r^{2-\alpha}\right) e^{-2 O\left(r^{2-\alpha}\right)} \leq C,
$$

namely, $\rho \leq O\left(e^{2 r_{\rho}}\right)$. This shows that our result is more general than Lam's results Lam.

\section{Weighted $p$ Poincaré INEQUality}

In this section, we study the space of harmonic $p$-forms on manifolds with property $\mathcal{P}_{p, \rho}$. The curvature operator $K_{p}$ on $M$ is assumed to satisfy the inequality

$$
K_{p} \geq-\frac{m-p+1}{m-p} \rho(x)
$$

for all $x \in M$. In this theorem, a growth assumption is needed for the weighted function of the Poincaré type inequality. For the sake of completeness, we restate the following decay estimate in [C-S] (cf. D] and [L-W2]). 
Lemma 3.1. Let $M$ be a complete noncompact manifold. If $E$ is an end of $M$ with the property $\left(\mathcal{P}_{p, \rho}\right)$ for some nonnegative weight function $\rho$, then for any smooth harmonic $p$ form $w$ satisfying

$$
\int_{E_{\rho}(2 R) \backslash E_{\rho}(R)} \rho(x) \exp \left(-2 r_{\rho}\right)|w|^{2} d v=o(R),
$$

we have

$$
\int_{E_{\rho}(R)} \rho(x) \exp \left(2 r_{\rho}\right)|w|^{2} d v \leq C R
$$

and

$$
\int_{E_{\rho}(R+1) \backslash E_{\rho}(R)} \rho(x) \exp \left(2 r_{\rho}\right)|w|^{2} d v \leq C,
$$

for all $R$ sufficiently large and for some constant $C$ depending on $w$ and $\rho$.

Theorem 3.2. Let $M$ be a complete noncompact manifold with property $\left(\mathcal{P}_{p, \rho}\right)$ for some nonnegative weight function $\rho$. Assume that the curvature operator $K_{p}$ on $M$ has the lower bound

$$
K_{p} \geq-\frac{m-p+1}{m-p} \rho(x)
$$

and $\rho \leq O\left(e^{2 r_{\rho}}\right)$. Suppose that $\omega$ is a harmonic $p$-form in $L^{2}(p \geq 2)$. Then either

(1) $H^{p}\left(L^{2}(M)\right)=0$ or

(2) there exists a 1-form $\alpha$ with $\alpha \wedge \omega=0$ such that

$$
\nabla \omega=\alpha \otimes \omega-\frac{1}{m-p+1} \sum_{j=1}^{m} \theta^{j} \otimes\left(\theta^{j} \wedge i_{\alpha \# \omega}\right),
$$

where $\left\{\theta^{1}, \ldots, \theta^{m}\right\}$ is an orthonormal basis for the cotangent bundle and $a^{\#}$ is the dual vector of $\alpha$.

Proof. The proof is similar to the proof of Theorem 1.6. Suppose that $\omega \neq 0$. First, we claim that

$$
\int_{M} \rho|\omega|^{2}<\infty
$$

Indeed, since $\rho \leq O\left(e^{2 r_{\rho}}\right)$ and $|\omega| \in L^{2}(M)$, for any $R>1$, we have

$$
\int_{B_{\rho}(2 R) \backslash B_{\rho}(R)} \rho e^{-2 r_{\rho}}|\omega|^{2} \leq C
$$

for some constant $C$. By Lemma 3.1, we have

$$
\int_{B_{\rho}(R+1) \backslash B_{\rho}(R)} \rho|\omega|^{2} \leq C e^{-2 R} .
$$

Hence,

$$
\begin{aligned}
\int_{B_{\rho}(2 R) \backslash B_{\rho}(R)} \rho|\omega|^{2} & \leq \sum_{r=[R]}^{2[R]+1} \int_{B_{\rho}(r+1) \backslash B_{\rho}(r)} \rho|\omega|^{2} \\
& \leq \sum_{r=[R]}^{2[R]+1} C e^{-2 r} \leq 2 C[R] e^{-2[R]}
\end{aligned}
$$


Since $\sum_{n=1}^{\infty} 2^{n}[R] e^{-2^{n}[R]}$ converges,

$$
\int_{M} \rho|\omega|^{2}=\int_{B(R)} \rho|\omega|^{2}+\sum_{n=1}^{\infty} \int_{B_{\rho}\left(2^{n} R\right) \backslash B_{\rho}\left(2^{n-1} R\right)} \rho|\omega|^{2}<\infty .
$$

The claim is proved. Now, by the Bochner type formula for harmonic $p$-forms (see C-S] ) and the curvature assumption, we have

$$
h \Delta h \geq-\frac{(m-p+1) \rho(x)}{m-p} h^{2}+\frac{1}{m-p}|\nabla h|^{2} .
$$

Note that in this formula, we have $a(x)=\frac{(m-p+1) \rho(x)}{m-p}, b=\frac{1}{m-p}$. By (5) and (11), $h$ has a finite Dirichlet integral. Let $\phi$ be a cut-off function such that

$$
\phi= \begin{cases}1, & \text { on } B(R), \\ 0, & \text { on } M \backslash B(2 R)\end{cases}
$$

and $|\nabla \phi|^{2} \leq \frac{C}{R^{2}}$ on $B(2 R) \backslash B(R)$.

By (5),

$$
\int_{M}|\nabla h|^{2} \leq \frac{1}{\frac{1}{m-p}+1} \int_{M} \frac{(m-p+1) \rho(x)}{m-p} h^{2}=\int_{M} \rho(x) h^{2} .
$$

Hence $\int_{M} \rho(x) h^{2}=\int_{M}|\nabla h|^{2}$ for $M$ has property $\left(\mathcal{P}_{p, \rho}\right)$.

If we multiply both sides of (12) by $\phi$ and integrate it over $M$, the divergence theorem gives

$$
-\int_{M}\langle\nabla(\phi h), \nabla h\rangle \geq-\frac{m-p+1}{m-p} \int_{M} \phi \rho(x) h^{2}+\frac{1}{m-p} \int_{M} \phi|\nabla h|^{2} .
$$

Now, letting $R \rightarrow \infty$ and using the fact that $h \in L^{2}(M),|\nabla h| \in L^{2}(M)$,

$$
-\int_{M}|\nabla h|^{2} \geq-\frac{m-p+1}{m-p} \int_{M} \rho(x) h^{2}+\frac{1}{m-p} \int_{M}|\nabla h|^{2} ;
$$

consequently,

$$
-\int_{M} \rho(x) h^{2} \geq-\frac{m-p+1}{m-p} \int_{M} \rho(x) h^{2}+\frac{1}{m-p} \int_{M}|\nabla h|^{2} .
$$

This implies

$$
\int_{M} \rho(x) h^{2} \geq \int_{M}|\nabla h|^{2} .
$$

Hence all of the preceding inequalities become equalities. In particular, inequality (12) is an equality, i.e.,

$$
h \Delta h=-\frac{(m-p+1) \rho(x)}{m-p} h^{2}+\frac{1}{m-p}|\nabla h|^{2} .
$$

By a similar argument in $\mathrm{C}-\mathrm{S}$, the proof is complete.

Now we focus on a complete manifold that satisfies both a weighted $p$-Poincaré inequality and the curvature operator lower bound. A similar vanishing result for harmonic forms (see [L-W1, Lam]) is given as follows. 
Theorem 3.3. Let $M^{m}$ be a complete noncompact Riemannian manifold with property $\left(\mathcal{P}_{p, \rho}\right)$ for some nonnegative weight function $\rho$. Assume that $\rho \leq O\left(e^{2 r_{\rho}}\right)$ and the curvature operator $K_{p}$ of $M$ has the lower bound

$$
K_{p} \geq-\left(\frac{m-p+1}{m-p} \rho-\epsilon\right)
$$

for some given $\epsilon>0$. Then $\operatorname{dim} H^{p}\left(L^{2}(M)\right)=0$.

Proof. Let $\omega$ be a harmonic $p$-form in $L^{2}(M)$ and $h=|\omega|$. By the argument given in the proof of Theorem 1.7, we have that

$$
\int_{M} \rho|\omega|^{2}<\infty
$$

Moreover, we have the Bochner formula

$$
h \Delta h \geq-\left(\frac{m-p+1}{m-p} \rho-\epsilon\right) h^{2}+\frac{1}{m-p}|\nabla h|^{2} .
$$

By (5),

$$
\int_{M}|\nabla h|^{2} \leq \frac{1}{\frac{1}{m-p}+1} \int_{M}\left(\frac{m-p+1}{m-p} \rho-\epsilon\right) h^{2}=\int_{M} \rho(x) h^{2}-\epsilon \int_{M} h^{2} .
$$

Since $h \in L^{2}$ and $\int_{M} \rho h^{2}<\infty$, it implies $|\nabla h| \in L^{2}(M)$. Note that $M$ has $\left(\mathcal{P}_{p, \rho}\right)$ property; hence $M$ also has a weighted Poincaré inequality with the nonnegative weight function $\rho$. Combining this with the $L^{2}$ property as above, we infer that

$$
\int_{M} \rho h^{2} \leq \int_{M}|\nabla h|^{2}
$$

Thus,

$$
\int_{M}|\nabla h|^{2} \leq \int_{M} \rho(x) h^{2}-\frac{m-p}{m-p+1} \epsilon \int_{M} h^{2} \leq \int_{M}|\nabla h|^{2}-\frac{m-p}{m-p+1} \epsilon \int_{M} h^{2} .
$$

This yields $\int_{M} h^{2}=0$; consequently $h=0$ or equivalently $\omega=0$. The proof is complete.

In the following theorem, we study the space of an $L^{2}$ harmonic function with finite energy of $M$ which satisfies a weighted Poincaré inequality with a nonnegative weight function $\rho(x)$. By Theorem 2.1 in $[\mathrm{D}-\mathrm{S}$, we have

$$
\int_{B_{\rho}(R+1) \backslash B_{\rho}(R)} \rho u^{2} \leq C e^{-2 R} .
$$

By a similar argument in Theorem 2.2 the splitting and vanishing properties are established on $M$.

Theorem 3.4. Let $M^{m}$ be a complete noncompact Riemannian manifold with prop$\operatorname{erty}\left(\mathcal{P}_{p, \rho}\right)$ for some nonnegative weight function $\rho$. Assume that the Ricci curvature satisfies

and $\rho \leq O\left(e^{2 r_{\rho}}\right)$, Then either

$$
\operatorname{Ric}_{M}(x) \geq-\frac{m}{m-1} \rho(x)
$$

(1) $H\left(L^{2}(M)\right)=\mathbb{R}$ or

(2) $M=\mathbb{R} \times N$ with warped metric product, where $N$ is a manifold of dimension $(m-1)$. 


\section{ACKNOWLEDGMENTS}

The first author would like to express his gratitude to NSC, Taiwan, for a valued three-year scholarship through the National Tsinghua University. A part of this work was done when the first author attended the KAWA2 workshop in Marseille, France. He would like to thank Professor Thomas Pascal for the kind invitation and financial support.

\section{REFERENCES}

[C-G] Mingliang Cai and Gregory J. Galloway, Boundaries of zero scalar curvature in the AdS/CFT correspondence, Adv. Theor. Math. Phys. 3 (1999), no. 6, 1769-1783 (2000). MR:1812136 (2002k:53080)

[C-S] Jui-Tang Ray Chen and Chiung-Jue Anna Sung, Harmonic forms on manifolds with weighted Poincaré inequality, Pacific J. Math. 242 (2009), no. 2, 201-214, DOI 10.2140/pjm.2009.242.201. MR2546710 (2010k:53122)

[D] Harold Donnelly, Eigenforms of the Laplacian on complete Riemannian manifolds, Comm. Partial Differential Equations 9 (1984), no. 13, 1299-1321, DOI 10.1080/03605308408820364. MR764665 (86c:58145)

[D-S] N. T. Dung and C. J. Sung, Smooth metric measure spaces with weighted Poincaré inequality, Math. Z. 273 (2013), no. 3-4, 613-632. DOI 10.1007/s00209-012-1023-y. MR3030670

[Lam] Kwan-Hang Lam, Results on a weighted Poincaré inequality of complete manifolds, Trans. Amer. Math. Soc. 362 (2010), no. 10, 5043-5062, DOI 10.1090/S0002-9947-10-04894-4. MR2657671 (2011f:53062)

[Li] P. Li, Harmonic functions and applications to complete manifolds, "Lecture Notes" given at the University of California, Irvine, 2004. Available on the author's webpage.

[L-W1] Peter Li and Jiaping Wang, Complete manifolds with positive spectrum, J. Differential Geom. 58 (2001), no. 3, 501-534. MR1906784 (2003e:58046)

[L-W2] Peter Li and Jiaping Wang, Weighted Poincaré inequality and rigidity of complete manifolds, Ann. Sci. École Norm. Sup. (4) 39 (2006), no. 6, 921-982 (English, with English and French summaries), DOI 10.1016/j.ansens.2006.11.001. MR2316978(2008d:53053)

[Wang] Xiaodong Wang, On conformally compact Einstein manifolds, Math. Res. Lett. 8 (2001), no. 5-6, 671-688. MR.1879811 (2003d:53075)

[W-Y] Edward Witten and S.-T. Yau, Connectedness of the boundary in the AdS/CFT correspondence, Adv. Theor. Math. Phys. 3 (1999), no. 6, 1635-1655 (2000). MR.1812133 (2002b:53071)

Department of Mathematics, National Tsing Hua University, Kuang-Fu Road, HsinCHU, TAIWAN 30013

E-mail address: dungmath@yahoo.co.uk

Department of Mathematics, National Tsing Hua University, Kuang-Fu Road, HsinCHU, TAIWAN 30013

E-mail address: cjsung@math.nthu.edu.tw 